\title{
Modeling two-dimensional structure at the core-mantle boundary
}

\author{
D. V. Helmberger \\ Seismological Laboratory, California Institute of Technology, Pasadena
}

\author{
E. J. Garnero \\ Earth Sciences Department, Institute of Tectonics, University of California, Santa Cruz
}

\author{
X. Ding \\ Seismological Laboratory, California Institute of Technology, Pasadena
}

\begin{abstract}
Recent studies of $S K S$ waveform modeling emphasize the strong variation of seismic properties at the core-mantle boundary (CMB) and the need for two-dimensional and three-dimensional waveform modeling capabilities. In particular, the bifurcation of $S K S$ into $S P_{d} K S$ and $S K P_{d} S$ near $110^{\circ}$ shows strong regional variations. The first of these phases has a $P$ wave diffraction along the bottom of the mantle near the source, while the latter phase occurs at the receiver end. Generalized ray theory proves effective in generating theoretical seismograms in this type of problem because each of these diffractions is associated with a particular transmission coefficient: $T_{s p}$ which transmits shear waves into primary waves when crossing the CMB and $T_{p s}$ which transmits the primary waves back into shear waves at the receiver end. Each region can then be isolated and have its separate fine structure, sharp or gradational. Two classes of boundaries are explored: the CMB as a simple, sharp interface and the CMB with a very low velocity transition layer (10\% slower than reference models). The two diffractions produced by these structures have diagnostic arrival times and wave shapes and when combined with the geometric $S K S$ produce distinct waveform characteristics not easily generated by other means. Since the ray paths associated with these three phases are virtually identical in the mantle and only differ along a short sample of $\mathrm{CMB}$ and in the one-dimensional fluid core, we can isolate the small localized CMB region sampled. Thus the waveform character of the extended $S K S$ in the range of $105^{\circ}$ to $120^{\circ}$ becomes an excellent CMB probe which we demonstrate on a small sample of observations from the Fiji-Tonga region as recorded in North America.
\end{abstract}

\section{Introduction}

Recently, Garnero et al., [1993] emphasized the use of the waveform distortion of $S K S$ near its bifurcation with $P$ wave diffraction along the core-mantle boundary as a means of studying seismic structure near this boundary. Figure 1 displays the geometric ray paths associated with these phases along with their corresponding waveform predictions from the reflectivity method [Kind and Müller, 1975]. As discussed by Choy [1977], the geometric $S K S$ phase has a zero near $107^{\circ}$ for global average reference models, such as preliminary reference Earth model (PREM) [Dziewonski and Anderson, 1981]. At this distance the transmission coefficient of $S$ to $P\left(T_{s p}\right)$ across the CMB goes to zero, and the energy is reflected back into the mantle as a $P$ wave. This $P$ wave travels along the CMB interface diffracting energy into the core. At larger distances, $S K S$ begins to separate from $S P_{d} K S$ (diffraction on the source end) and $S K P_{d} S$ (diffraction on the receiver end) because of its smaller ray parameter. As is apparent from Figure 1, the core-mantle crossing points of $S K S$ and $S P_{d} K S$ are very close

Copyright 1996 by the American Geophysical Union.

Paper number 96JB00534.

0148-0227/96/96JB-00534\$09.00 together all the way out to $125^{\circ}$ where they are still less than $300 \mathrm{~km}$ apart [Garnero et al., 1993] (hereinafter referred to as GGH). Thus any distortions in the interference between these phases is likely to be associated with mantle-side CMB structure, where the $P_{\text {diff }}$ occurs. Results from GGH suggest that many observations favor stronger, more delayed diffractions than can be explained by PREM-type models.

Reflectivity synthetics from their preferred models are displayed in Figure 2 along with PREM predictions for comparison. These models have a 5\% reduction in $P$ velocity at the mantle base tapered to PREM at 50 and $100 \mathrm{~km}$ up from the CMB. Such models delay $S P_{d} K S$ and $S K P_{d} S$ relative to $S K S$, enhancing amplitudes of the diffracted pulse, as suggested in many observations. Subsequent analysis of these core phases provides strong evidence for lateral variation. Thus it is likely that $S K P_{d} S$ and $S P_{d} K S$ are not encountering the same structure [Gamero and Helmberger, 1995]. This paper addresses an adaptation of the generalized ray method to treat such situations.

Previous studies have used the notation $S P_{d} K S$ to denote the diffraction that occurs at either the source or receiver sides of the path. In this paper, however, we utilize two-dimensional (2-D) models having lowermost mantle structure on the source side of wave paths different from that on the receiver side. In 

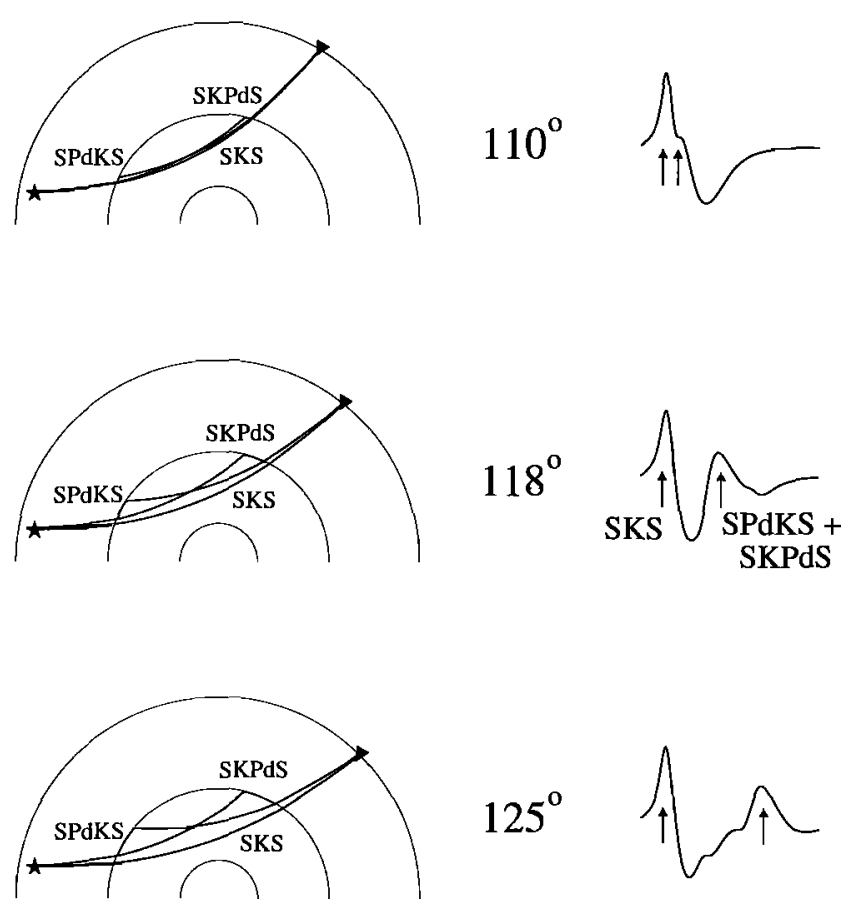

$125^{\circ}$

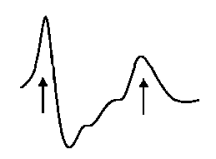

$35 \mathrm{~s}$

Figure 1. Cross sections at three ranges $\left(110^{\circ}, 118^{\circ}\right.$, and $\left.125^{\circ}\right)$ showing the ray paths of $S K S, S P_{d} K S$, and $S K P_{d} S$ assuming the P-5-50 model and associated synthetics.

such modeling experiments the source-side and receiver-side diffractions are affected differently, even becoming separate phases at large distances. Therefore we utilize the notation $S P_{d} K S$ and $S K P_{d} S$ to separately denote diffraction on the source and receiver sides of the path, respectively.

\section{Method}

One of the commonly used methods of generating body wave synthetic seismograms is from Chapman [1976], called the WKBJ method. This method is discussed and tested in a review paper by Chapman and Orcutt [1985] for onedimensional (1-D) layered models, including core phases. A modification of the method based on an earlier Chapman [1974] paper is discussed for 2-D core models more recently by Helmberger et al. [1995]. In this paper we return to the basic Cagniard-de Hoop method and investigate the rather complex behavior of these $P$ diffractions associated with $S K S$. This procedure requires considerable computational effort, since complex ray parameters are involved, but it is good to start with a well-known method to address new problems before making approximations, as suggested by the above authors.

The general Cagniard-de Hoop method is discussed in detail in many books [e.g., Aki and Richards, 1980]. The usual procedure is to break the velocity-depth function into layers and to perform an Earth-flattening approximation [Gilbert and Helmberger, 1972; Müller, 1977]. The wave field in terms of generalized rays can be approximated by summing the primary rays only;

$$
\phi(x, t)=\sqrt{\frac{2}{x}} \frac{1}{\pi}\left(\frac{\Delta}{\sin \Delta}\right)\left[\frac{1}{\sqrt{t}} * \sum \Psi_{j}(p)\right]
$$

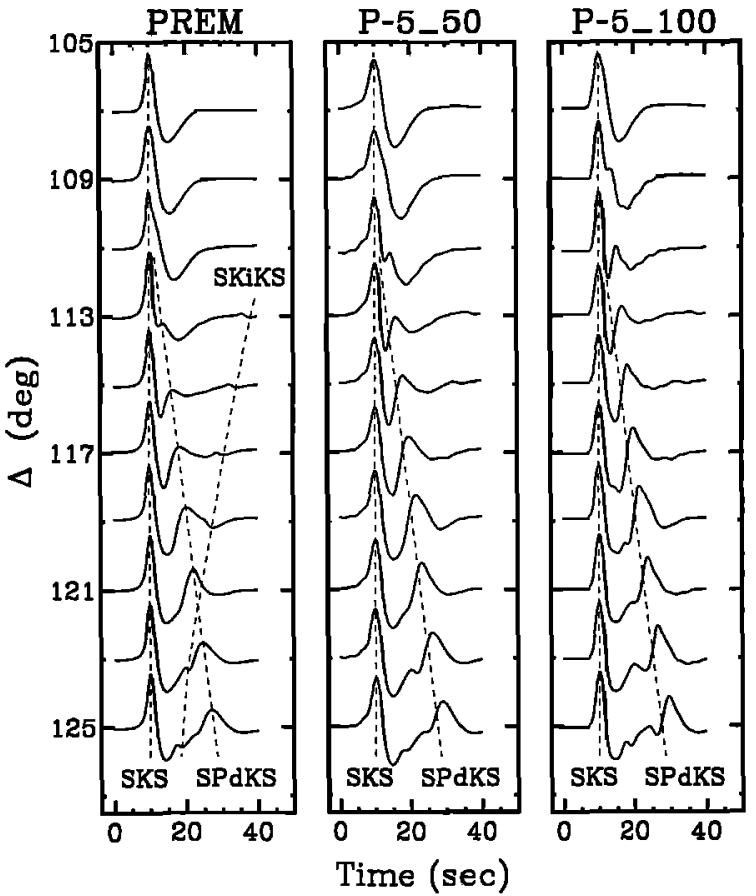

Figure 2. Three columns of synthetics corresponding to PREM: a 5\% reduction at the CMB tapered to PREM $50 \mathrm{~km}$ above the CMB and a $5 \%$ reduction at the CMB tapered to 100 $\mathrm{km}$ above the CMB [after Garnero et al., 1993].

where $x$ is the receiver distance, $t$ is time after origin, and $\Delta$ is the epicentral distance in radians. The $(\Delta / \sin \Delta)$ factor corrects for a spherical Earth as discussed above with accuracy demonstrated by Helmberger [1973], where

$$
\Psi,(p) \equiv \operatorname{Im}\left(\frac{\sqrt{p}}{\eta_{1}} \Pi(p) \frac{d p}{d t}\right)_{J}
$$

and $p$ is ray parameter or slowness.

The function $d p / d t$ is obtained from

$$
t=p x+2 \sum_{j=1}^{n} \mathrm{Th}_{j} \eta_{j}
$$

where $p(t)$ forms the complex contours $\eta_{j}=\left(1 / \alpha_{j}^{2}-p^{2}\right)^{1 / 2}$ [Helmberger, 1983]. The layer thicknesses are given by $\mathrm{Th}$, and velocities are given by $\alpha$. Note that for each interface we must determine a $p=p_{0}$ and associated $\iota_{0}$ which describe a path connecting the interface $j$ to the source and receiver satisfying Snell's law. The factor $\Pi(p)$ contains the product of all the

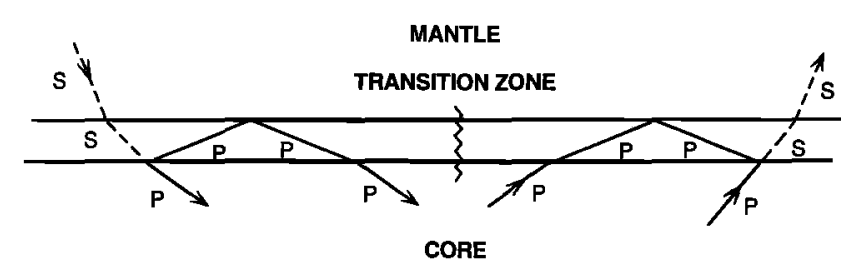

Figure 3. Schematic ray diagram indicating the multiple reflections that can develop at the CMB when signals enter the layer as $S V$, penetrate the core as $P$, and return. 


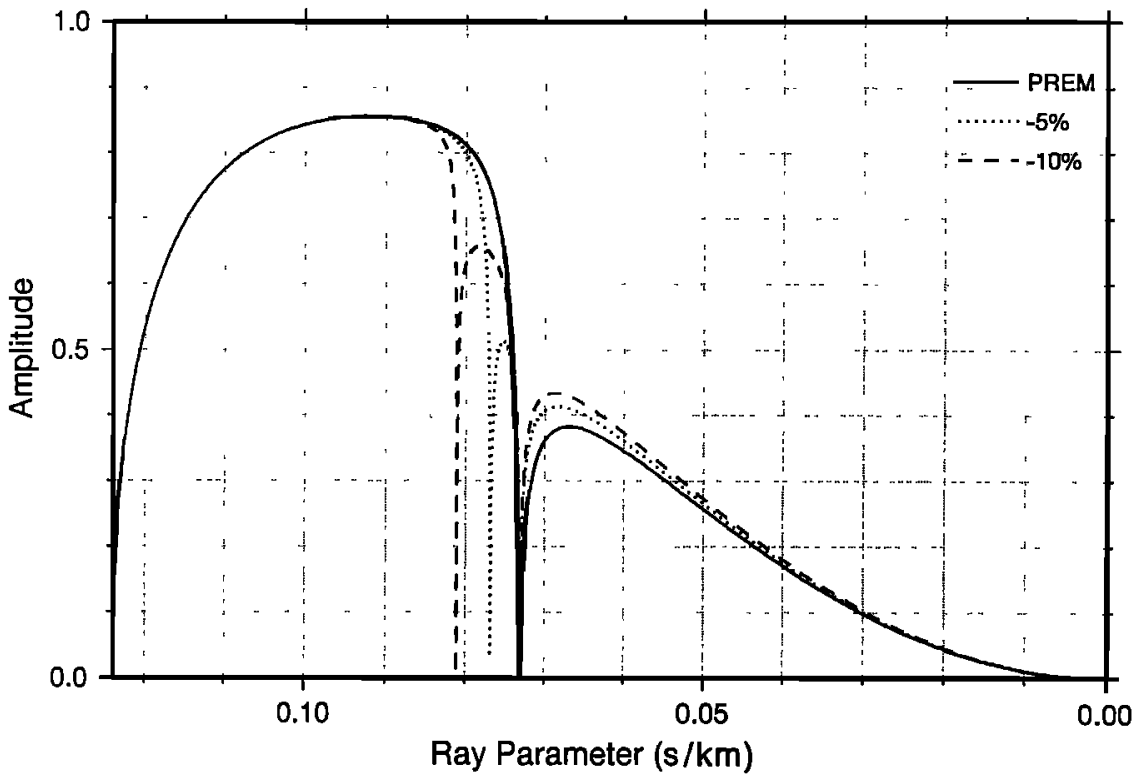

Figure 4. Plot of ray parameter vs. geometric amplitude which contains the product of the real part of $\left(T_{s p} T_{p s}\right)$ for three models: PREM at both ends, PREM at one end with a $5 \%$ reduction at the other end, and PREM at one end with a $10 \%$ reduction at the other end.

transmission and reflections encountered by ray $j$. In this application we will break these up into three groups:

$$
\Pi(p)=\Pi_{p}(p) \Pi_{c}(p) \Pi_{r r}(p) .
$$

The first set, $\Pi_{p}$, contains the product of all the $S$ wave transmission coefficients from the source down to the core and back up to the receiver. The second set, $\Pi_{c}$, contains the product of all the $P$ wave transmission coefficients and reflection coefficients along the ray path in the core. The third set, $\Pi_{t r}(p)$, contains the coefficients associated with crossing the CMB region. For a sharp interface model, $\Pi_{t r}(p)$ is simply the product of $T_{s p}(p)$ and $T_{p s}(p)$, where $T_{s p}$ describes the transmission coefficient appropriate for a mode-change $S V$ to $P$ on the source end and similarly for $T_{p s}$ on the receiver end. When we include a transition layer, the various other coefficients must be included as indicated in Figure 3. The convergence of this ray series depends on the velocity jumps and the nature of this transition layer as discussed below.

We have neglected the effects of source radiation and receiver functions in this discussion because they can be considered constant over the range of interest. They can be easily included as discussed by Helmberger [1983]. With these simplifications a synthetic seismogram can be calculated by performing the following convolutions:

$$
Q(x, t)=d / d t[\phi(x, t) * I(t) * S(t) * A(t)]
$$

where $I(t), S(t)$, and $A(t)$ represent the functions describing the instrumental response, the source time history, and the attenuation operator [Helmberger and Burdick, 1979].

\section{CMB as a Sharp Interface}

We begin with a simple calculation with no CMB transitional layer assuming the velocity model PREM. The details of Earth flattening for PREM and a comparison of core phases against full wave theory [Choy, 1977] are discussed in detail by Song and Helmberger [1992]. In this particular application the model has been interpolated into 20 -km-thick layers. Following (1), we need to sum generalized rays over these interfaces to generate $S K S$ and its associated diffractions. Note that when evaluating this expression for simple problems, the response obtained from (1) will be a step-like function which turns on at
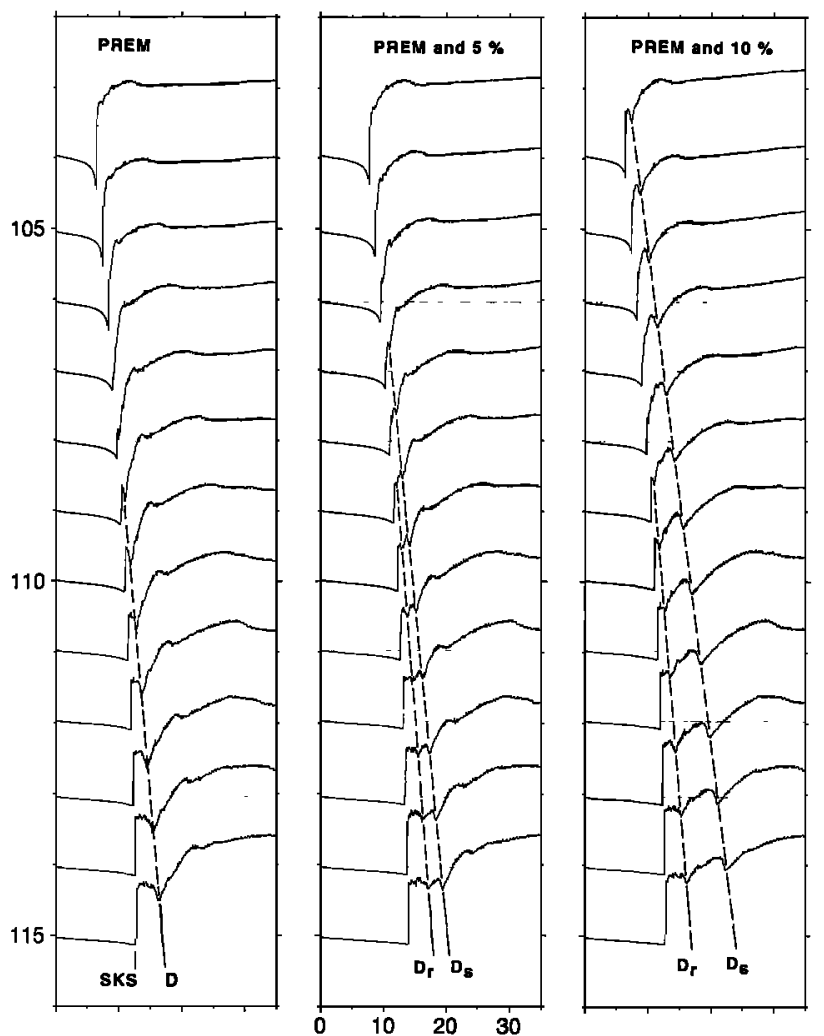

Figure 5. Comparison of step responses for the three test cases displaying $S K S$ and diffractions indicated by the dashed lines; $D_{r}\left(S K P_{d} S\right), D_{s}\left(S P_{d} K S\right)$, and $D\left(S K P_{d} S+S P_{d} K S\right)$. 

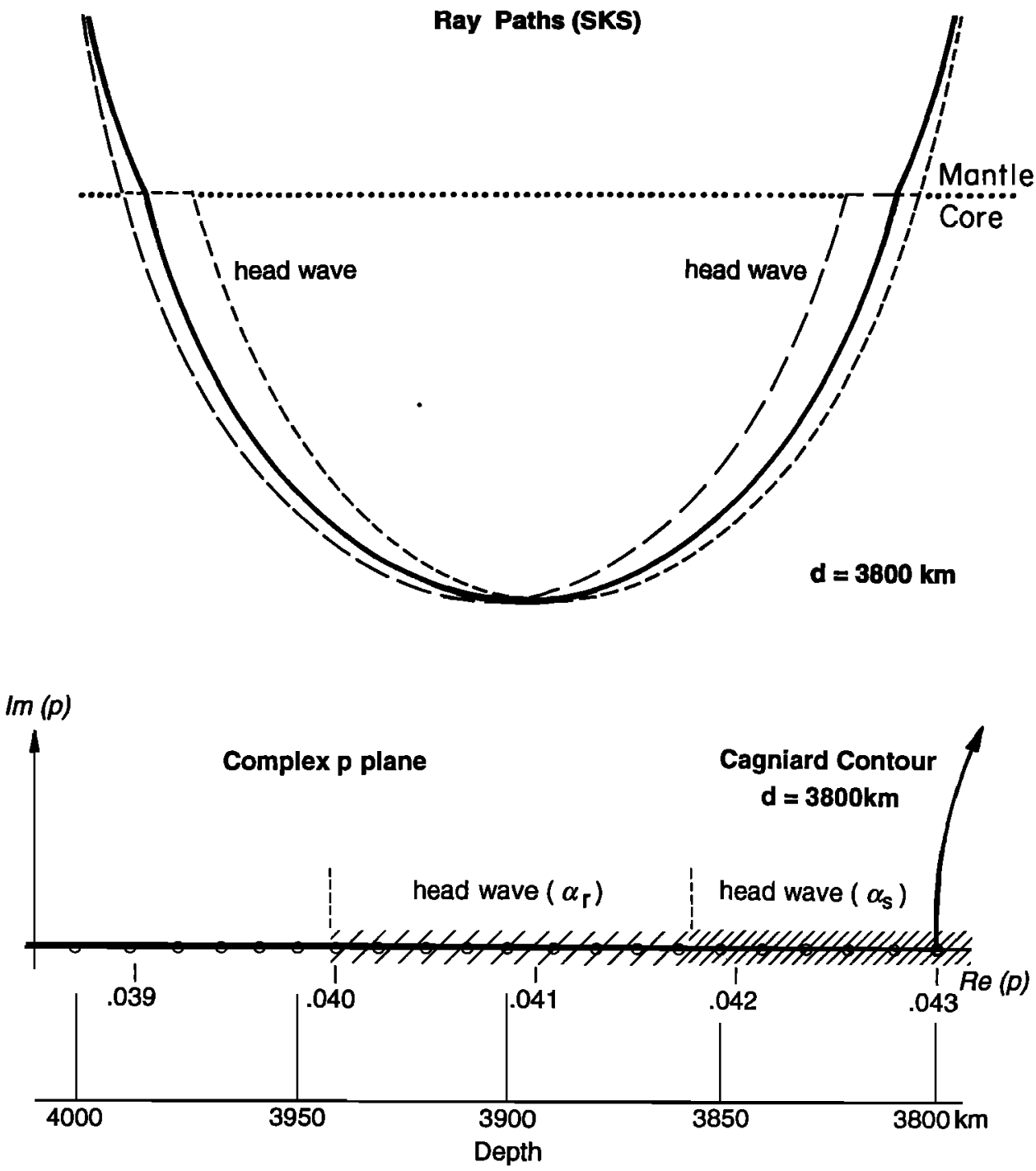

Figure 6. (top) Paths appropriate for $S P_{d} K S$ and $S K P_{d} S$ in PREM. (bottom) These paths lose their symmetry for laterally varying models when the branch cuts become distinct. The Cagniard contour displayed is associated with a ray bottoming at a depth of $3800 \mathrm{~km}$. The small open circles indicate the $p_{0}$ associated with the other generalized rays contributing to the two diffracted pulses. The two diffractions $S P_{d} K S$ and $S K P_{d} S$ are associated with the two branch cuts $\left(d_{r}\right)$ and $\left(d_{s}\right)$, respectively.

the arrival time and decays according to geometrical spreading [Helmberger, 1983]. Thus $S K S$ should appear as a step, since it is a geometric phase, while diffractions should be less sharp in nature. These features are controlled by the transmission coefficients across the CMB, essentially $T_{s p}$ and $T_{p s}$. The real part of the product of these two factors, $\Pi_{t r}(p)$, is plotted for PREM in Figure 4, showing the zero at $p_{\epsilon}=1 / 13.7 \mathrm{~km}$ or when the ray parameter reaches the reciprocal of the $P$ velocity at the CMB. Included in this plot are $\Pi_{t r}(p)$ for the cases when the $P$ velocities are reduced at one end by $5 \%$ and $10 \%$. This asymmetry produces two critical angles where ray paths can become parallel to the CMB, one associated with $T_{s p}$ on the source end and one associated with $T_{p s}$ on the receiver end. For $p$ smaller than $p_{c}$, the product $\Pi_{t r}(p)$ is real and relatively small. Thus at ranges larger than $110^{\circ}$ the $S K S$ phase returns to the receiver looking like the source or a step shape as is apparent at $115^{\circ}$ in Figure 5. The effective ray parameter for the geometric arrival is greater than $p_{c}$ at ranges greater than $104^{\circ}$, and the product of the coefficient becomes complex.
This means that $S K S$ is no longer a true minimum phase and has a small negative precursor [Helmberger, 1983].

This feature can be understood in the generalized ray formalism in terms of the position of the branch cuts relative to those real ray parameters dominating the response (Figure 6). The critical $p_{c}$ is about 0.04 in Earth-flattened coordinates, so that for the larger ranges $p<p$, and the branch cuts have little effect. For $p$ much larger than $p$, the tip of the branch cuts are remote, and their influence is again slight. Only when the $p_{0}$ are near the branch cut tips, where $p \approx p_{c}$, will the transmission coefficients be rapidly varying and diffractions become important.

An example of a ray path appropriate for the generalized ray reflecting at a depth of $3800 \mathrm{~km}$, near $900 \mathrm{~km}$ beneath the $\mathrm{CMB}$, is displayed in the top panel of Figure 6 with the corresponding Cagniard-de Hoop path given in the bottom panel. The heavy line indicates the path controlled by Snell's law or $p_{0}=0.043$. The other two lines display the paths taken by the two head waves or diffractions associated with this particular 

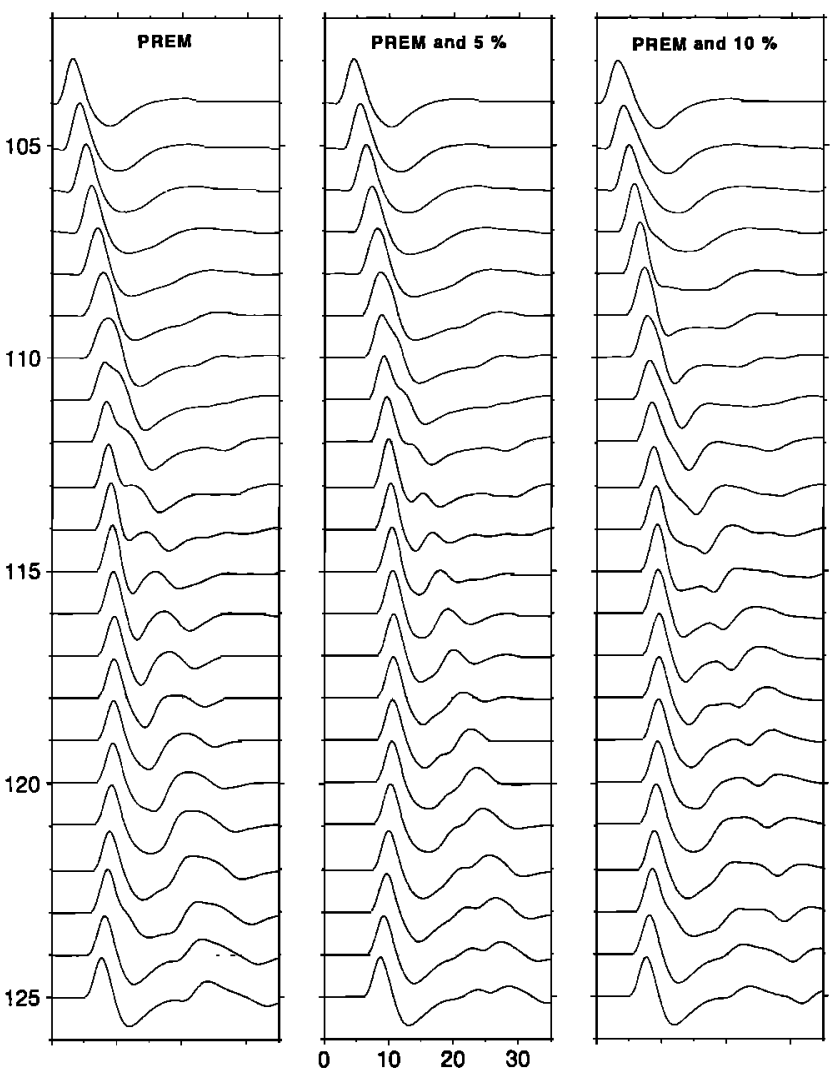

Figure 7. Comparison of synthetics for the three test cases displayed in Figure 5. These synthetics contain a trapezoidal time history $(1,1,1)$, a $t^{*}=1$, and a WWSSN long-period response. These parameters are the same as assumed by Garnero et al. [1993] and the reflectivity synthetics presented in Figure 2.

$p_{0}$. To break the symmetry, we assume that the $P$ velocity is reduced by $5 \%\left(\alpha_{s}\right)$ for $T_{s p}$ and the PREM velocity $\left(\alpha_{r}\right)$ for $T_{p s}$. The two branch cuts separate and form two diffractions as displayed in the bottom panel of Figure 6. In this case, their paths become asymmetric with a longer head wave path (along the $\mathrm{CMB}$ ) associated with the slower source-side velocity. The dashed lines indicate the two pulses $\left(S K P_{d} S\left(D_{r}\right)\right.$ and $S P_{d} K S\left(D_{s}\right)$ ) that have constant apparent velocities, $\alpha_{s}$ and $\alpha_{r}$. PREM shows the strongest diffraction because these two arrivals have the same travel times. This is also apparent in the corresponding synthetics displayed in Figure 7. These synthetics were generated by taking a time derivative of the above step responses and convolving with a long-period world-wide instrument response attenuation and source as expressed in (5). The synthetics for PREM are nearly identical to those generated by reflectivity (see Figure 2) except at the largest distance where the interference caused by $S K i K S$ has been neglected in the generalized ray theory (GRT) synthetics. All the synthetics presented here and by GGH assumed the same attenuation operator $\left(t^{*}=1\right)$ and time histories given by a symmetric trapezoid (1 s, $1 \mathrm{~s}, 1 \mathrm{~s})$.

The three columns of synthetics displayed in Figure 7, while similar, are different in two important features. First, the strong shoulder that appears in the PREM waveforms at $111^{\circ}$ and $112^{\circ}$ is less apparent in the other two cases. Second, when the two basal velocities differ by over $5 \%$, their resulting diffractions become distinct at the larger distance as is apparent in Figure 7. However, the thickness of the bottommost layer has an obvious effect which we address next.

\section{CMB With a Transition Layer}

Adding a low-velocity zone at the base of the mantle has a strong effect on the rate of $S P_{d} K S$ decay with range as demonstrated by GGH. The physical reason for this can be understood by examining the nature of head waves. As the head wave travels along the $\mathrm{CMB}$, it is radiating energy both upward and downward. Thus it is decaying faster than a body wave which is the case of all diffracted waves. When a low-velocity zone is added at the base of the mantle, it turns energy back downward which tends to strengthen the diffraction. Thus the downgoing energy $S P_{d} K S$ decays more slowly with range. These features are quite apparent in the ray synthetics when we break the ray contributions up as in Figure 8 where a transitional layer is assumed beneath the source region. The model beneath the receiver region is assumed to be PREM.

The rays summed here are those introduced earlier in Figure 3 assuming a $40-\mathrm{km}$ layer with a $10 \%$ drop in velocity. The individual traces are plotted on the same amplitude scale as the bottom sum (trace e). Thus the largest contribution comes from the ray crossing the transition layer as $S$ wave and entering the core $S(d)$. This is the response obtained in the previous section; that is,

$$
\Pi_{l r}(p)=T_{s s}^{s} T_{s p}^{s} T_{p s}^{r},
$$

where $T$ denotes the TC of $S$ wave to $S$ wave at the top of the transition layer, and the other two factors contain the usual $S K S$ interaction. The earliest response is produced by the ray crossing the layer as $P$ (trace a) and entering the core. In this case,

$$
\Pi_{t r}(p)=\left(T_{s p}^{s} T_{p p}^{s}\right) T_{p s}^{r}
$$

where the superscript $s$ refers to the source end and $r$ refers to the receiver end. Trace $b$ contains the response of a $P P$ bounce in the layer where

$$
\Pi_{t r}(p)=\left(T_{s s}^{t} R_{s p}^{b} R_{p p}^{t} T_{p p}^{b}\right) T_{p s}^{r}
$$

In this expression we are using the superscript to denote the interaction at the bottom of the layer as $b$ and at the top as $t$. The $R_{s p}^{b}$ coefficient can be large near $p=1 / \alpha_{s}$, where $\alpha_{s}$ is the compressional velocity of the layer. This emphasizes low-angle reflections. The $R_{p p}^{t}$ can have a critical angle in that the velocity above the layer is faster than the layer velocity. The response for $\Delta=108^{\circ}$ is actually precritical angle, while $\Delta=$ $120^{\circ}$ is postcritical angle. The long-period downswing corresponds to a $P$ head wave traveling along the top of the layer before traveling down into the core. Trace $c$ contains trace $b$ plus the various multiples. The latter rays contribute little and appear to be in the numerical noise. The larger the velocity drop at the top of the layer the larger this response, since it is controlled mainly by the transmission coefficient $T_{s p}^{s}$.

Profiles of these types of responses as a function of layer thickness are given in Figure 9. The phase labeled $D_{r}$ is the same in all three profiles, since it is produced at the sharp interface on the receiver end of the path. It is essentially $S K P_{d} S$. The phase labeled $D_{s}$ is more complicated, since it no longer has a constant phase velocity, and the line is simply the approximate arrival which does depend on the layer thickness. When the layer is thin, $5 \mathrm{~km}$, the phase $D_{s}$ approaches values that are appropriate for a sharp interface (see PREM results in 


$$
\Delta=108
$$

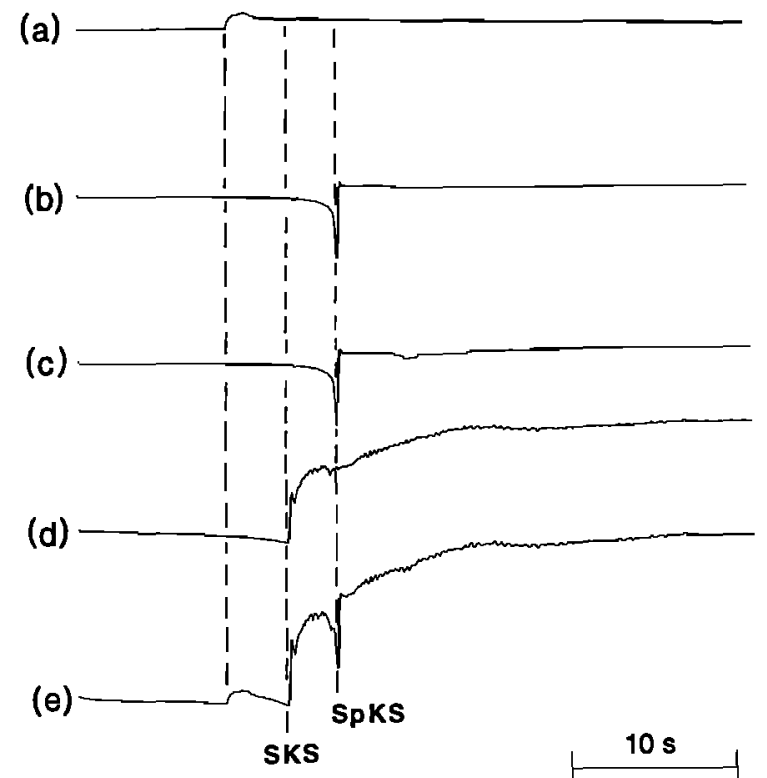

$\Delta=120$

Figure 8. Construction of the step response in terms of rays at distances of $108^{\circ}-120^{\circ}$. Traces a and $\mathrm{d}$ contain the step responses of the generalized rays crossing the layer as a $P$ wave and $S$ wave, respectively. Trace b displays the response of ray reflecting one time in the layer as a $P$ wave. Trace c contains $S P P$ plus the other possible mode changes $S P S, S S P, S S S$ and $P P P, P P S, P S P$, and $P P P$. Trace e contains the sum of a, c, and d. The amplitudes are normalized to the lower trace e.

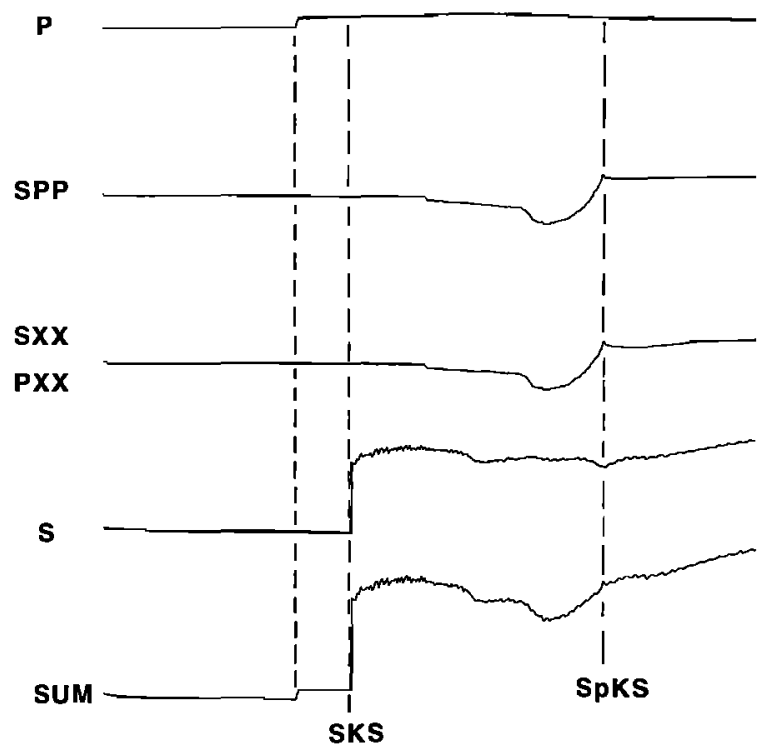

Figure 5). The responses become difficult to distinguish when $l$ reaches values that are less than about $2 \mathrm{~km}$.

The phase $S P P$ is particularly interesting in that it has a critical angle near $107^{\circ}(l=40 \mathrm{~km})$, where the generalized ray reflecting back down from the top of the transition layer begins to develop a head wave. This wave travels along the top of the transition layer radiating head waves back down toward the core. At larger ranges the head wave tends to catch up to the $S P_{d} K S$ arrival since it has a faster phase velocity. It has the same phase velocity as $S K P_{d} S$. As the layer becomes thinner, the critical angle develops more quickly and arrives near the $S K P_{d} S$. Thus at long periods the synthetics for the $5-\mathrm{km}$ case are nearly the same as for PREM except that they are shifted by about $1^{\circ}$ as can be seen by comparing columns on the left in Figures 7 and 10.

The synthetics displayed in the various columns of Figure 10 share many characteristics. For example, at ranges less than about $113^{\circ}$ one can overlay the synthetics on the left with about a $2^{\circ}$ shift of those on the right; that is, $111^{\circ}(5 \mathrm{~km})$ compares with $109^{\circ}(20 \mathrm{~km})$ and $107^{\circ}(40 \mathrm{~km})$, etc. At larger ranges the shift is less, but the separation between $S K S$ and its diffractions increases considerably faster for the thicker layers. Thus comparing these waveforms with observations becomes an effective means of investigating the CMB structure as we address next.

\section{Application and Discussion}

In this section we will use the above synthetics to explain a few observations as a demonstration of their usefulness. As suggested earlier, the properties of these synthetics are somewhat similar to those in the 1-D study (GGH), and thus we will
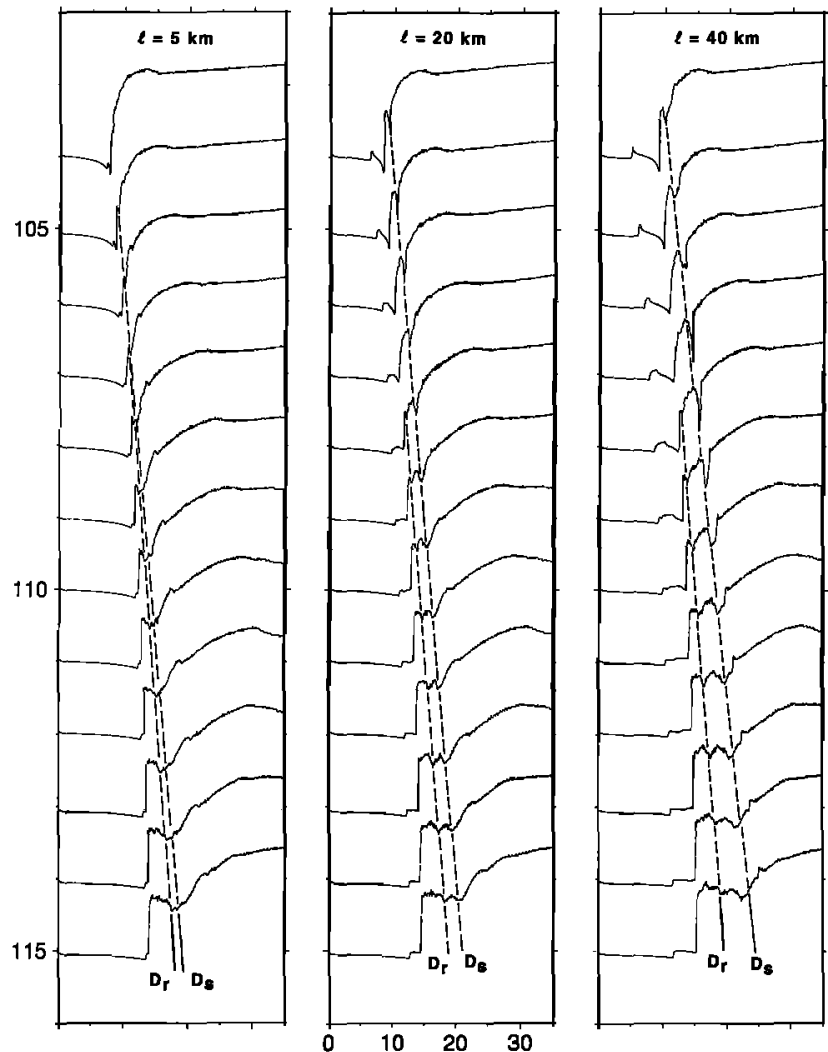

Figure 9. Step responses for the three test cases with layer thicknesses of 5,20 , and $40 \mathrm{~km}$. The dashed lines indicate the two diffractions: $D_{r}\left(S K P_{d} S\right)$ and $D_{s}\left(S P_{d} K S\right)$. 
briefly revisit some of the same data. Figure 11 presents the paths and associated World-Wide Standard Seismograph Network (WWSSN) long-period data from their January 24, 1969 event along with 2-D synthetics. The $S P_{d} K S$ segments underlay the mid-Pacific low-velocity structure [e.g., Su and Dziewonski, 1994] except possibly to SJG which could miss the anomaly. The data contain a pronounced double arrival at AAM and BLA indicative of the interference between $S K S$ and $S P_{d} K S$ which is typical of southwestern Pacific events recorded at these stations. As presented by GGH, synthetics from PREM produce this effect but at a greater distance, roughly a $4^{\circ}-5^{\circ}$ shift. These anomalous observations relative to PREM are for the same azimuth to the North American stations as the most anomalous $S-S K S$ and $S K K S-S K S$ data presented by Garnero and Helmberger [1995, 1996] and Garnero et al. [1993]. Apparently, $S P_{d} K S$ paths are sampling the large-scale mid-Pacific structure as reported by $\mathrm{Su}$ and Dziewonski [1994], Masters et $a l$. [1992], and others. The receiver-side $D_{r}$ or $S K P_{d} S$ paths are sampling beneath the United States which correspond with faster than average shear $D^{\prime \prime}$ velocities [Grand, 1994]. Mantle basal velocities in $P$ also appear to be high as reported by Garnero and Helmberger [1996] and Wysession et al. [1992]. Moreover, the recent broadband studies by Ding and Helmberger [1996] suggest that while the shear velocities are explained adequately by the Lay-type models [Lay and Helmberger, 1983], PREM explains the $P$ waveform and travel times very well. This conclusion is supported by short-period stacked data as well [Mori and Helmberger, 1995]. Thus we will assume PREM on the receiver side and allow the source-side paths to vary.
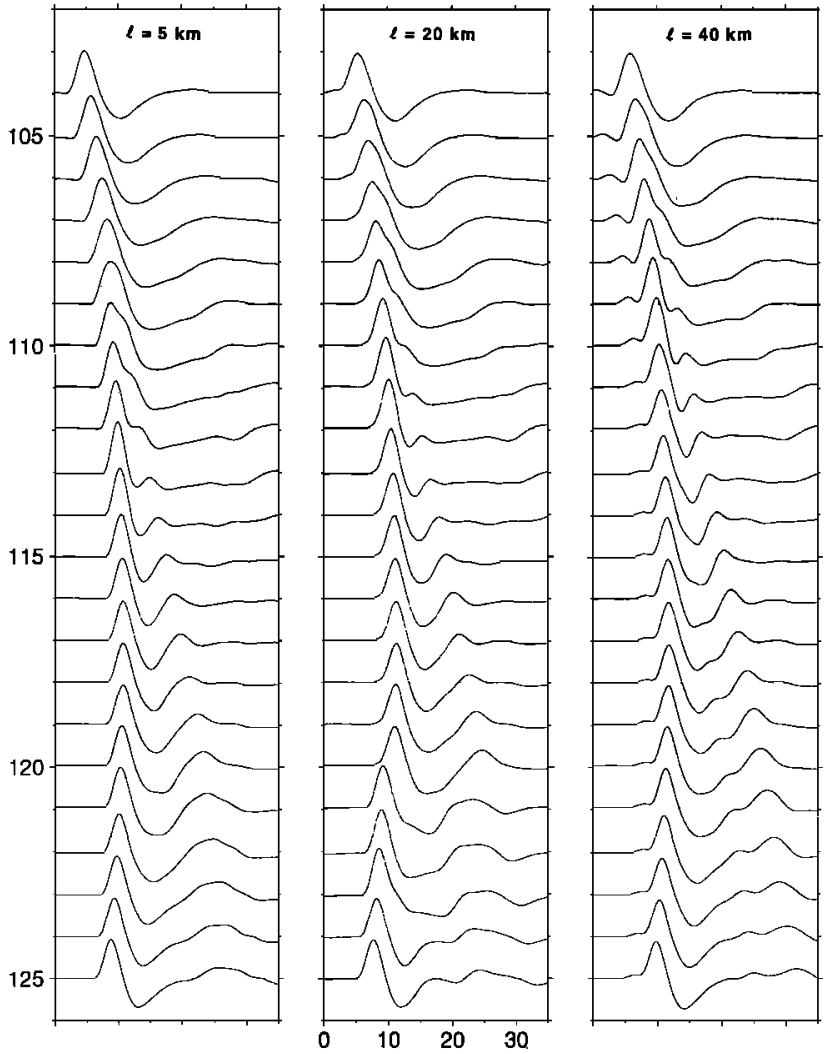

Figure 10. Synthetics corresponding to the step responses displayed in Figure 9 for the three test cases with layer thicknesses of 5,20 , and $40 \mathrm{~km}$.
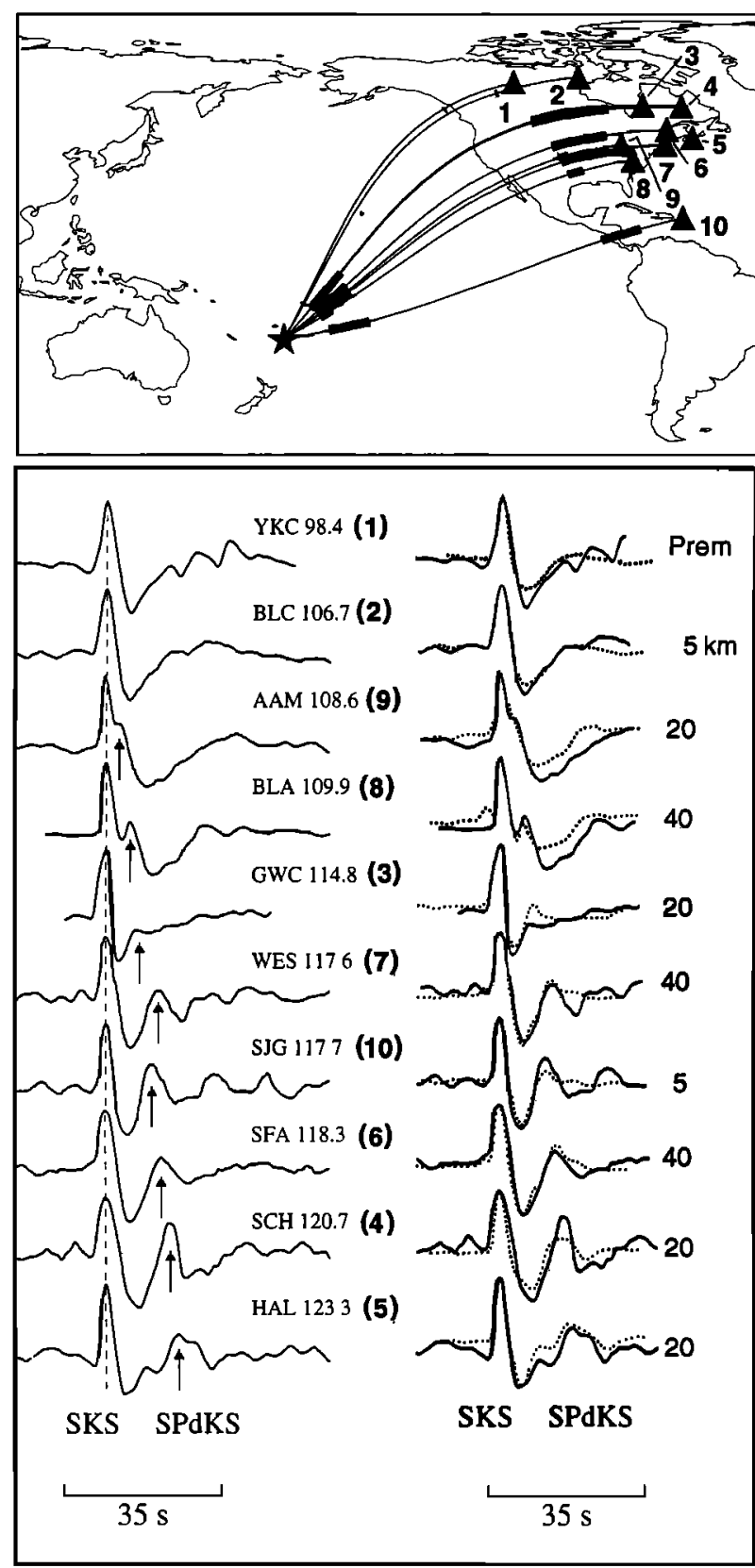

Figure 11. (top) Paths appropriate for the various stations: YKC and BLC are the most northern two and SJG is the most southern; the numbers indicate the various locations of the Standard Worldwide Stations identified by the three letters. The other cluster of paths appears to sample the strongest anomaly. (bottom) Data on the left with synthetics indicated by dotted lines superimposed on data indicated by solid lines on the right.

The synthetics displayed on the right in Figure 11 were selected from the profiles presented earlier in Figure 10 with the layer thickness as the variable. Although these fits are not perfect, they appear to capture the general character of the observations. Note that we have allowed the transition layer to vary from 5 to $40 \mathrm{~km}$ which appears to be an effective means of moving the $D_{s}$ relative to $S K S$. Although the selected thicknesses vary considerably, they do seem to group with only the path to SJG (10) being distinctly different. Note that the fits before $107^{\circ}$ (YKC and BLC) could just as well be to any of the 

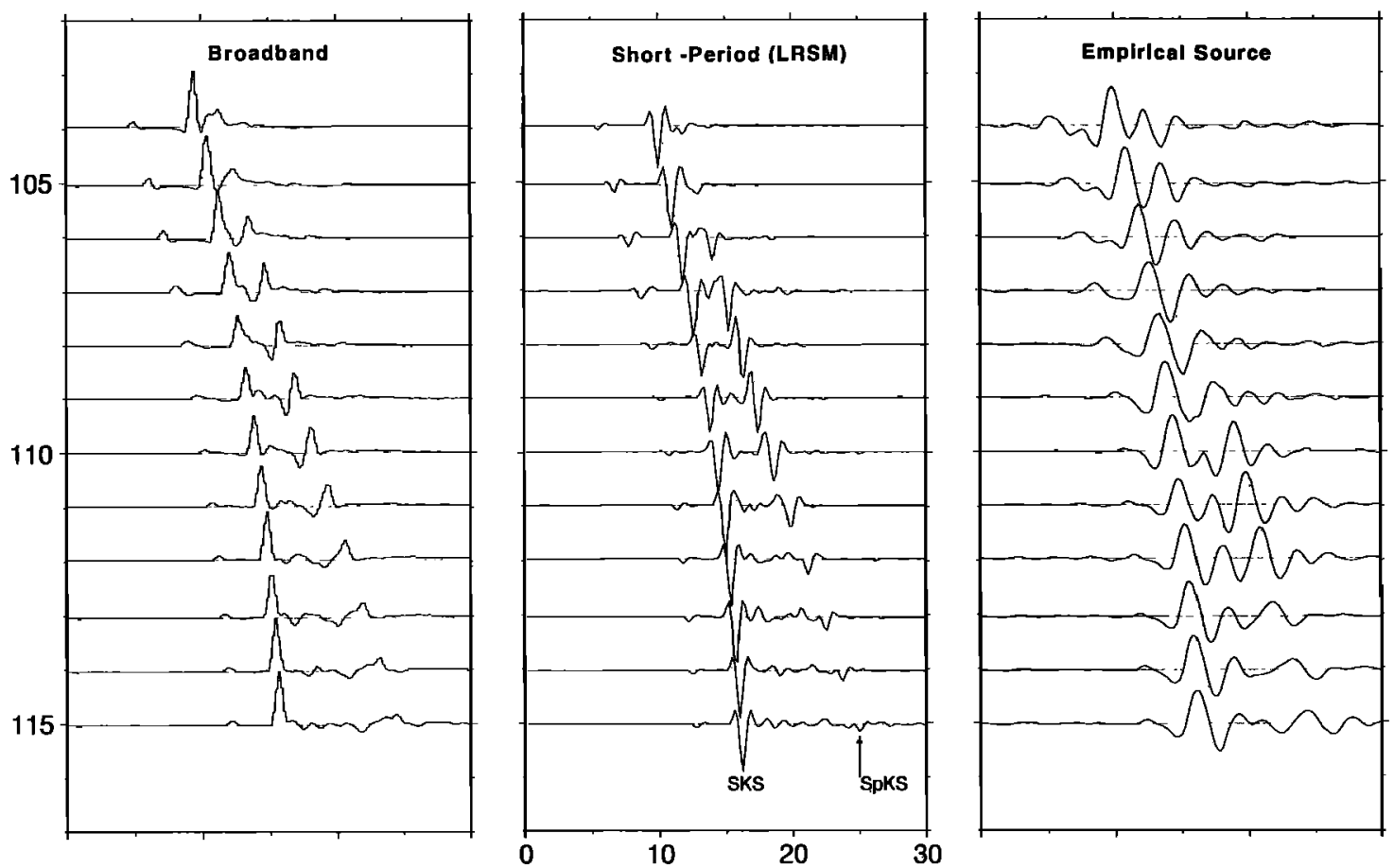

Figure 12. Synthetics displaying the relatively strong $S_{p} K S$ phase at ranges $107^{\circ}-110^{\circ}$.

above layered models, since the synthetics are about the same as displayed in Figure 10. Although the timing is quite good in overlay comparisons, the relative strength of the diffractions is difficult to explain in some records. There is always the difficulty in defining the strengths of secondary arrivals in the presence of complex receiver structure [i.e., Zhang and Lay, 1984]. Corrections are possible by examining these stations at other ranges where only $S K S$ is arriving, for example $90^{\circ}-105^{\circ}$, and making adjustments as proposed by Zhang and Lay.

One way to obtain higher resolution and expand the data set is to explore the use of short-period observations as reported on recently by Silver and Bina [1993]. Figure 12 displays synthetics for the $l=40 \mathrm{~km}$ case in terms of broadband displacement (left), standard WWSSN short-period synthetics (middle), and empirical source function synthetics (right). The development of a strong secondary arrival between $106^{\circ}$ and $109^{\circ}$ is quite clear which corresponds to the phase $S P P$ passing through critical angle (see Figure 8 ). Since this pulse is totally reflected back down into the core, it becomes essentially a geometric phase which we will call $S_{p} K S$. Its geometric amplitude compared to $S K S$ is displayed in Figure 13. Figure 13 compares the real part of the product of $\left(T_{s p} T_{p s}\right)$ assuming

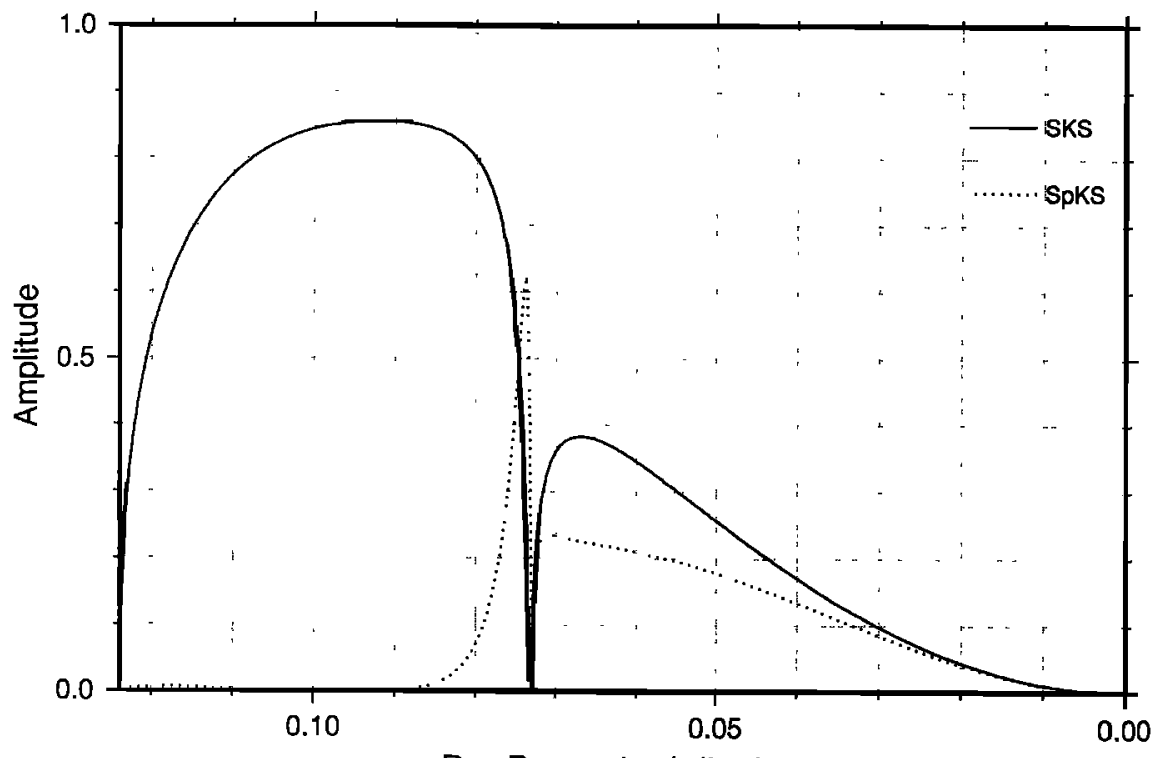

Ray Parameter (s/km)

Figure 13. Plot of geometric amplitude versus ray parameter for $S K S$ and $S_{p} K S$ assuming PREM. 
PREM and the real part of the product of $\left(R_{s}, T_{p p} T_{p s}\right)$, where $R_{s p}$ is the reflection of $S V$ to $P$ at the CMB. The reflection $P$ to $P$ back down from the top boundary as stated in (8) is assumed to be near one or geometric in nature. Thus the phase $S_{p} K S$ can be relatively large over a small window of ray parameter since $R_{, p}$ and $R_{p p}$ can be large. Since it appears that the thickness of the transition zone varies, we can expect to see considerable variation in the strength of this phase.

Unfortunately, the high attenuation of short-period shear waves coupled with source complexity makes it difficult to observe such detail as suggested earlier by Choy [1977]. However, it may be possible with modern broadband arrays and the right geometry to reach this resolution. One reason for optimism is displayed in Figure 14. Figure 14 displays long-range seismic measurement (LRSM) analog records from a Kermadec Island event (August 5, 1964, $h=216 \mathrm{~km}$ ). About 25 LRSM stations recorded this event with most measurements made in the western United States. A cluster of seven recordings (Tonto Forest Array, see Figure 14a) provides a clear
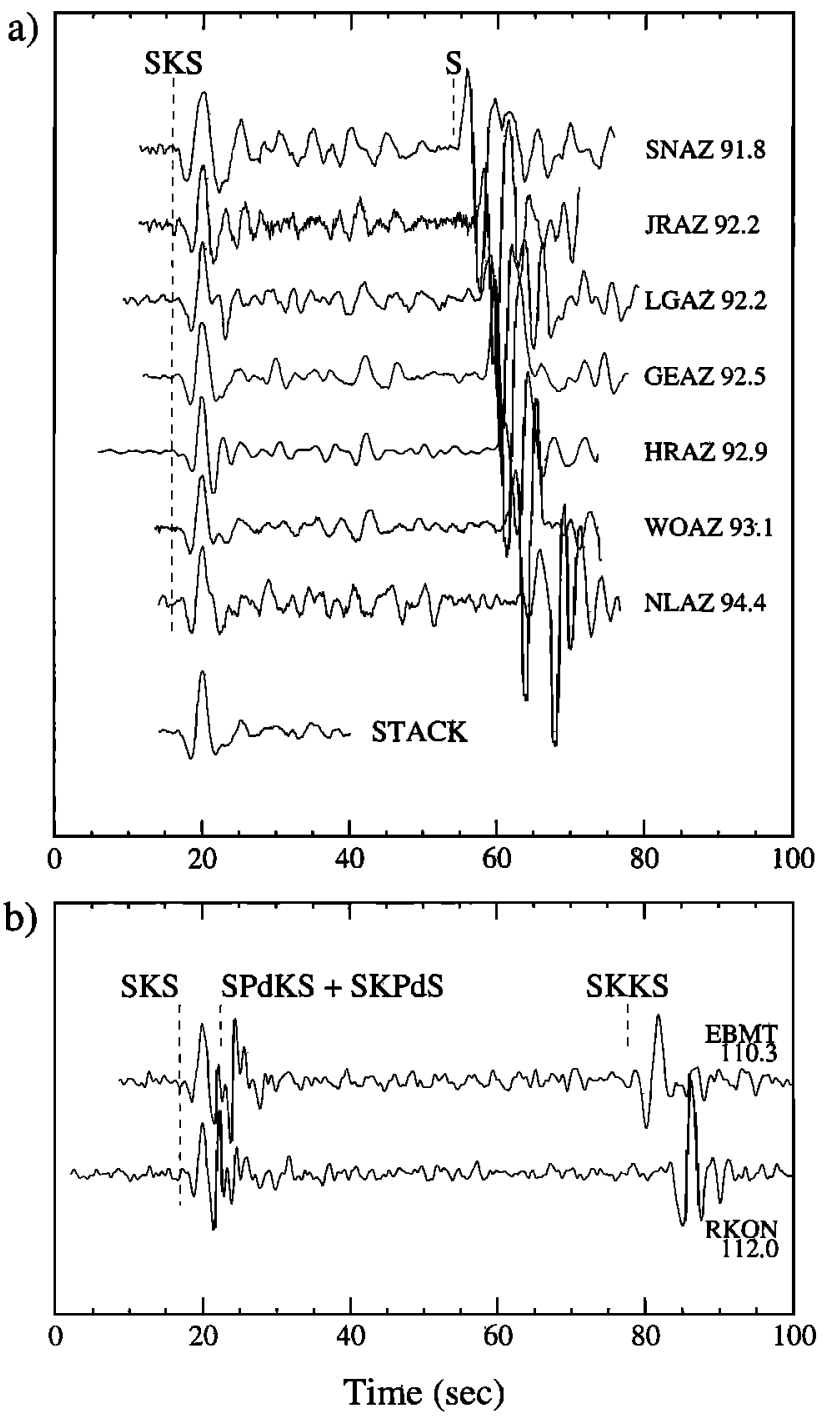

Figure 14. LRSM data at stations forming (a) the Tonto Forest Array and (b) two stations in Canada, East Braintree, Manitoba (EBMT), and Red Lake, Ontario (RKON), of the Kermadec event.

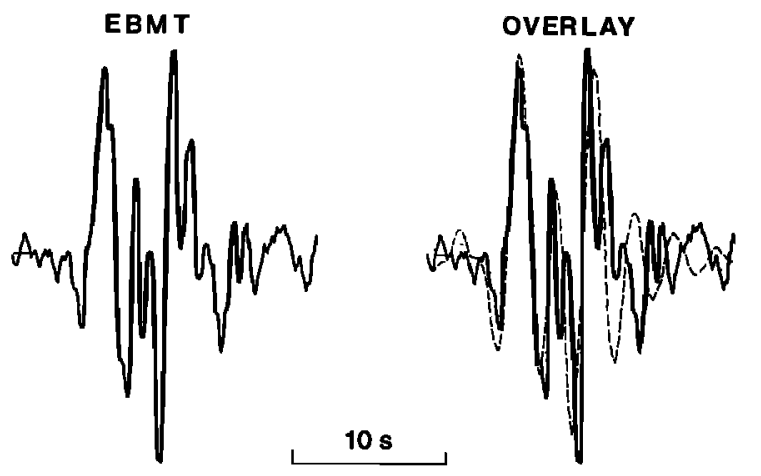

Figure 15. A comparison of the EBMT observation with synthetic overlay indicated by dashed lines. The synthetic is the same as given in Figure 12 at $110^{\circ}$, assuming the $40-\mathrm{km}$ layer with a $10 \%$ drop in velocity.

sample of the source function. A stack of these waveforms defines the empirical source function used in generating the synthetics in Figure 12 (right). These synthetics can be compared directly with the observed waveforms of Figure 14b.

The secondary arrival observed at East Braintree, Manitoba (EBMT) compares quite well with those in Figure 12. An enlarged view of EBMT is given in Figure 15 along with a synthetic overlay for comparison. The fit is quite good except that it has somewhat higher frequency. Since EBMT and Red Lake, Ontario (RKON) are on the edge of a shield, this feature is predicted [i.e., Lay and Helmberger, 1981]. Secondary arrivals on short-period records are quite common and can easily be caused by complex receiver structure. However, $S K K S$ does not show such complexity. Moreover, the two stations EBMT and RKON have particularly simple receiver functions as established earlier by Helmberger and Wiggins [1971]. More data are required to be conclusive, but it appears that short-period and broadband data could be very useful in establishing the existence of $S_{p} K S$ and providing short-wavelength resolution of this particular transition zone.

\section{Conclusions}

This paper reviews the issue of generating synthetics for $S K S$ and the two associated diffractions, $S P_{d} K S$ and $S K P_{d} S$. While these diffractions are symmetric for one-dimensional models, they become distinct when 2-D models are addressed. If the variation is slight, it tends to reduce the effective interference with $S K S$ and to make even their detection difficult. In fact, most data do not show very dramatic interference which suggests that this is the norm; essentially, PREM with a few percent variation between the source and receiver crossings [Garmero and Helmberger, 1995]. This situation is supported by tomography models. However, some very anomalous data were recently reported by Garnero et al. [1993] for paths sampling beneath the mid-Pacific, and subsequent studies [Gamero and Helmberger, 1996] indicate that these strong diffractions are well correlated with anomalies in $S K K S-S K S, S_{\text {diff, }}$ etc. These patterns are also seen in tomographic studies where the Pacific is circled by normal to fast paths (PREM) [i.e., Su and Dziewonski, 1994]. A detailed broadband study of $P$ waveforms using all the stations along the west coast of North America [Ding and Helmberger, 1996] reached the same conclusion. This is substantiated by Mori and Helmberger [1995] using hundreds 
of stacked short-period data. They also report on similar stacks of Fiji data indicating a thin low-velocity layer at the CMB with a sharp top (5-10\% reduction). If we assume the PREM model beneath the United States (for $S K P_{d} S$ ), we obtain a similar result in modeling the $S K S-S P_{d} K S$ data. The existence of a low-velocity zone at the base of the mantle not only leads to enhanced long-period diffractions but also predicts the existence of a new geometric short-period arrival, $S_{p} K S$. Essentially, the $S$ to $P$ reflected energy $\left(S_{p}\right)$ at the CMB is turned back downward caused by the negative velocity gradient. This phase is predicted to occur at distances near $110^{\circ}$ and then only when sampling beneath lower mantle anomalies.

The modeling presented here suggests considerable lateral variation where the transition layer appears to vary from 5 to $40 \mathrm{~km}$. These samples are only a few hundred kilometers apart, so the changes are quite sharp. Such resolution must be questioned, but it makes other differential core phases $P K P(A B)$ $P K P(D F)$ sampling this same region easier to explain [i.e., Song and Helmberger, 1996]. In short, we are developing the tools and the data sets necessary to image these very interesting structures at the $\mathrm{CMB}$.

Acknowledgments. This research was supported by the National Science Foundation grant EAR-93-16441. We thank Don Anderson, Larty Burdick, Steve Roecker, and an anonymous referee for constructive reviews and Steve Grand, who first recognized these strange seismograms. This is contribution $\mathbf{5 5 3 0}$ of the Division of Geological and Planetary Sciences, California Institute of Technology.

\section{References}

Aki, K., and P. G. Richards, Quanttative Seismology, Theory and Methods, W. H. Freeman, New York, 1980.

Chapman, C. H., Generalized ray theory for an inhomogeneous medium, Geophys. J. R. Astron. Soc., 36, 673-704, 1974.

Chapman, C. H., Exact and approximate generalized ray theory in vertically inhomogeneous media, Geophys. J. R. Astron. Soc., 46, 201-233, 1976.

Chapman, C. H., and J. A. Orcutt, The computation of body wave synthetic seismograms in laterally homogeneous media, Rev. Geophys., 23, 105-163, 1985.

Choy, G. L., Theoretical seismograms of core phases calculated by frequency-dependent full wave theory, and their interpretation, Geophys. J. R. Astron. Soc., 51, 275-312, 1977.

Ding, X., and D. V. Helmberger, Modeling $\mathrm{D}^{\prime \prime}$ structure beneath Central America with broadband seismic data, Geophys. J. Int., in press, 1996.

Dziewonski, A. M., and D. L. Anderson, Preliminary reference Earth model (PREM), Phys. Earth Planet. Inter., 25, 297-356, 1981.

Garnero, E. J., and D. V. Helmberger, A very slow basal layer underlying the large-scale low-velocity anomalies in the lower mantle beneath the Pacific: Evidence from core phases, Phys. Earth Planet. Inter., 91, 161-176, 1995.

Garnero, E. J., and D. V. Helmberger, Seismic detection of a thin laterally varying boundary layer at the base of the mantle beneath the central Pacific, Geophys. Res. Lett., in press, 1996.

Garnero, E. J., S. P. Grand, and D. V. Helmberger, Low $P$ velocity at the base of the mantle, Geophys. Res. Lett., 20, 1843-1846, 1993.
Gilbert, F., and D. V. Helmberger, Generalized ray theory for a layered sphere, Geophys. J. R. Astron. Soc., 27, 57-80, 1972.

Grand, S. P., Mantle shear structure beneath the Americas and surrounding oceans, J. Geophys. Res., 99, 11,591-11,622, 1994.

Helmberger, D. V., Numerical seismograms of long-period body waves, from seventeen to forty degrees, Bull. Seismol. Soc. Am., 63, 633-646, 1973.

Helmberger, D. V., Theory and application of synthetic seismograms, in Earthquakes: Observation, Theory, and Interpretation, vol. 37, edited by H. Kanamori and E. Boschi, pp. 174-217, North-Holland, New York, 1983.

Helmberger, D. V., and L. J. Burdick, Synthetic seismograms, Annu. Rev. Earth Planet. Scl., 7, 417-442, 1979.

Helmberger, D. V., and R. A. Wiggins, Upper mantle structure of midwestern United States, J. Geophys. Res., 76, 3229-3245, 1971.

Helmberger, D. V., L.-S. Zhao, and E. J. Garnero, Construction of synthetics for 2-D structures; core phases, in Seismic Modeling of the Earth Structure, edited by E. Boschi, G. Ekstrom, and A. Morelli, North-Holland, New York, in press, 1996.

Kind, R., and G. Müller, Computations of $S V$ waves in realistic Earth models, J. Geophys., 41, 149-172, 1975.

Lay, T., and D. V. Helmberger, Body wave amplitude patterns and upper mantle attenuation variations across North America, Geophys. J. R. Astron. Soc., 66, 691-726, 1981.

Lay, T., and D. V. Helmberger, A lower mantle $S$-wave triplication and the shear velocity structure of $\mathrm{D}^{\prime \prime}$, Geophys. J. R. Astron. Soc., 75, 799-838, 1983.

Masters, G., H. Bolton, and P. Shearer, Large-scale 3-dimensional structure of the mantle (abstract), Eos Trans. $A G U, 73(14)$, Spring Meet. Suppl., 201, 1992.

Mori, J., and D. V. Helmberger, Localized boundary layer below the mid-Pacific velocity anomaly identified from a $P, P$ precursor, $J$. Geophys. Res., 100, 20,359-20,365, 1995.

Muiller, G., Earth-flattening approximation for body waves derived from geometric ray theory-improvements, corrections and range of applicability, J. Geophys., 42, 429-436, 1977.

Silver, P., and C. R. Bina, An anomaly in the amplitude ratio of $S K K S / S K S$ in the range $100^{\circ}-108^{\circ}$ from portable teleseismic data, Geophys. Res. Lett., 20, 1135-1138, 1993.

Song, X., and D. V. Helmberger, Velocity structure near the inner core boundary from waveform modeling, J. Geophys. Res., 97, 6573-6586, 1992.

Song, $\mathrm{X}$, and $\mathrm{D}$. V. Helmberger, $P K P$ differential travel times: Implications for three-dimensional lower mantle structure, Phys. Earth Planet. Inter., in press, 1996.

Su, W. J., and A. M. Dziewonski, Degree 12 model of shear velocity heterogeneity in the mantle, J. Geophys. Res., 99, 6945-6980, 1994.

Wysession, M. E., E. A. Okal, and C. R. Bina, The structure of the core-mantle boundary from diffracted waves, J. Geophys. Res., 97, 8749-8764, 1992

Zhang, J., and T. Lay, Investigation of a lower mantle shear wave triplication using a broadband array, Geophys. Res. Lett., 11, 620623, 1984.

X. Ding and D. V. Helmberger, Seismological Laboratory, 252-21, California Institute of Technology, Pasadena, CA 91125. (e-mail: helm@seismo.gps.caltech.edu)

E. J. Garnero, Earth Sciences Department, Institute of Tectonics, University of California, Santa Cruz, CA 95064.

(Received May 31, 1995; revised December 8, 1995; accepted February 13, 1996.) 